\title{
Centrifugal Distortion Constants in 1.3 Propiolactone
}

\author{
Luis Mario Boggia, Roberto Raul Filgueira *, and Oscar Manuel Sorarrain \\ Departamento de Física, Universidad Nacional de La Plata, Argentina. \\ Centro de Investigaciones Tecnológicas, U.T.N. La Plata, Argentina \\ and Damiano Damiani \\ Istituto Chimico "G. Ciamician"-Universita di Bologna e Laboratorio \\ di Spettroscopia Molecolare del C.N.R.-Bologna, Italia
}

(Z. Naturforsch. 29 a, 95-98 [1974]; received August 2, 1973)

\begin{abstract}
The centrifugal distortion constants for $\mathrm{O}-\mathrm{CH}_{2}-\mathrm{CH}_{2}-\mathrm{C}=\mathrm{O}$ were calculated using Dowling and Watson's formalisms. From the fitting analysis and the deviation of predicted transitions it is concluded that the second formalism must be adopted. For the different parameters we have: $A^{\prime}=12406.012 \pm 0.005 \mathrm{MHz} ; B^{\prime}=5244.456 \pm 0.002 \mathrm{MHz} ; \quad C^{\prime}=3869.191 \pm 0.002 \mathrm{MHz} ; \quad d_{J}=$ $-2.08 \pm 0.05 \mathrm{kHz} ; d_{J K}=-23.63 \pm 0.95 \mathrm{kHz} ; d_{K}=-33.43 \pm 1.69 \mathrm{kHz} ; d_{W J}=(0.56 \pm 0.01) \times 10^{-6}$; $d_{W K}=(5.08 \pm 0.22) \times 10^{-6}$.
\end{abstract}

\section{Introduction}

Though the theoretical treatments of centrifugal distortion effects in the rotational energy levels have been largely developed during the last 20 years ${ }^{1-14}$ only a few times have they been applied to actual cases ${ }^{15-36}$ and with only one ${ }^{34}$ ring molecule structure having been investigated. Most of the applications have dealt with light molecules $(3-4$ atoms) and as Kirchhoff ${ }^{37}$ has observed, very often it has been difficult to fit the observed spectra with a standard deviations equivalent to the expected measurement errors .

We have recently measured (or remeasured in some cases) the frequencies of many rotational

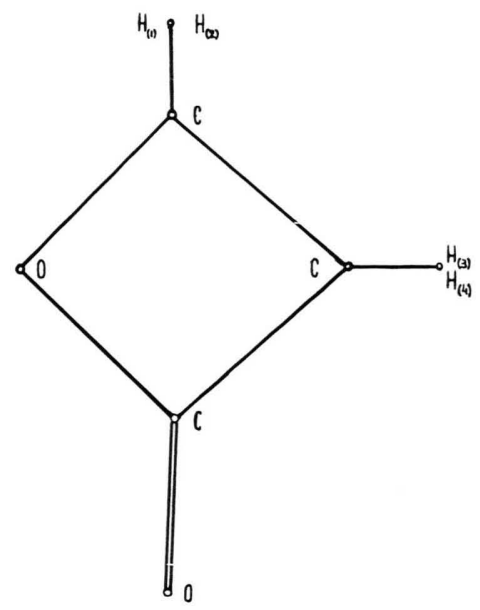

* Consejo Nacional de Investigaciones Científicas Técnicas, Argentina.

Reprint request to Departamento de Física (U.N.L.P.) Calles 49 y $115-$ C.C. 67, La Plata, Argentina. transitions for 1.3 Propiolactone ${ }^{38}$; this molecule is a strained four membered ring (see Fig. 1), where we have once more the opportunity to check the theory and experimental data. The principal features of this molecule are: $1^{\circ}$ ) heavier than many of the former molecules studied; $2^{\circ}$ ) a strained ring structure. Both characteristics lead us to suppose that we might find difficulties in evaluating the centrifugal distortion constants, but mainly because of their expected small values.

In this work we follow the procedures stated by Kirchhoff ${ }^{37}$ to get two sets of centrifugal distortion parameters: $1^{\circ}$ ) using the formalism of Dowling ${ }^{39}$; $2^{0}$ ) using the formalism of Watson ${ }^{11,13}$. From the following discussion we conclude that the second one must be adopted.

\section{Dowling Formalism}

Some workers have shown ${ }^{28-30}$ that Dowling's planar molecule constraints ${ }^{39}$ can be incorporated into the Kivelson-Wilson first order treatment to get four independent centrifugal distortion constants: $\tau_{x x x x}, \tau_{z z z z}, \tau_{x x z z}, \tau_{x z x z}$, even if the molecule is "almost" planar. In our case the molecule has planar symmetry and a small inertia defect, so we tried first this formalism. Experimental data were fitted by a least square treatment of equations of the form:

$$
\begin{gathered}
v_{i}-v_{i}{ }^{0}=\frac{\left(\partial v_{i}\right)^{0}}{\partial A} \Delta A+\frac{\left(\partial v_{i}\right)^{0}}{\partial B} \Delta B+\frac{\left(\partial v_{i}\right)^{0}}{\partial C} \Delta C \\
+\sum_{k} \frac{\left(\partial v_{i}\right)^{0}}{\partial \tau_{k}} \tau_{k}
\end{gathered}
$$


where $v_{i}$ are the observed frequencies, $v_{i}^{0}$ are rigid rotor frequencies calculated with a trial set of rotational constants $A^{0}-B^{0}, C^{0}$, and the $\tau_{k}$ are the distortion constants, as defined by Wilson and Howard ${ }^{1}$.

The fitting was achieved in several improved steps. In the first one, using together Boone's ${ }^{40}$ and our data, a set of rotational constants $A, B, C$ and $\tau_{k}$. were obtained. This set gave a standard deviation of $0.2 \mathrm{MHz}$, for the calculated frequencies. After remeasuring a few lines from Ref. ${ }^{40}$, we found an improved standard deviation of $0.15 \mathrm{MHz}$. The expected measurement errors, however, were estimated to be less than $0.05 \mathrm{MHz}$, so a procedure similar to the one suggested by Kirchhoff ${ }^{37}$ was tried. This procedure was made in three successive steps: $1^{0}$ ) a first fitting was achieved with all the lines with $J \leqq 10$ at our disposal. A sequential procedure was tested eliminating definitively from the fit (one at a time), those transitions which, when excluded from the fit, caused standard deviation to decrease; $2^{\circ}$ ) the procedure was repeated including all transitions with $J \leqq 20 ; 3^{\circ}$ ) again a new fitting was made including the remaining lines $(J \leqq 30)$.

The resulting constants can be seen in Table 1 . We can see that the sequential elimination proce- dure was not able to determine $\tau_{a a b b}$ and the total standard deviation increased slightly with the increasing $J$.

\section{Watson Formalism}

We next tried the Kivelson-Wilson formalism ${ }^{3}$ corrected by $\mathrm{W}$ atson ${ }^{11.13}$, where the general asymmetric top energies can be written as:

$$
\begin{aligned}
& W=W^{\prime}-d_{J} J^{2}(J+1)^{2}-d_{J K} J(J+1)\left\langle P_{z^{2}}\right\rangle \\
& -d_{K}\left\langle P_{z}{ }^{4}\right\rangle-d_{W J} W^{\prime} J(J+1)-d_{W K} W^{\prime}\left\langle P_{z}{ }^{2}\right\rangle
\end{aligned}
$$

where $W^{\prime}$ is the rigid rotor energy corresponding to a set of rotational constants $A^{\prime}, B^{\prime}, C^{\prime}$ which differ slightly from the effective rotational constants of Kivelson-Wilson formulation (see Reference ${ }^{11}$ ). Using again the sequential elimination in three steps, we found a more significant set of results (see Table 2). Here we can see that the inclusion of lines of higher $J$ gives a better determination for each constant, decreasing the total standard deviation in each step which is always less than the measurement error. In Table 3 we can see transitions used to calculate the rotational constants of Table 1 and Table 2 together with the eliminated

\begin{tabular}{|c|c|c|c|c|}
\hline \multicolumn{2}{|c|}{ Lines up to } & $J=10$ & $J=20$ & $J=30$ \\
\hline$A$ & $(\mathrm{MHz})$ & $12406.019 \pm 0.028$ & $12406.016 \pm 0.012$ & $12406.023 \neq 0.009$ \\
\hline$B$ & $(\mathrm{MHz})$ & $5244.445 \pm 0.007$ & $5244.453 \pm 0.006$ & $5244.457 \pm 0.004$ \\
\hline$C$ & $(\mathrm{MHz})$ & $3869.195 \pm 0.006$ & $3869.196 \pm 0.004$ & $3869.197 \pm 0.003$ \\
\hline$\tau_{\text {aaaa }}$ & $(\mathrm{kHz})$ & $-46.5 \pm 9.5$ & $-45.7 \pm 4.5$ & $-47.2 \mp 2.1$ \\
\hline$\tau_{b b b b}$ & $(\mathrm{kHz})$ & -2.9 & -3.8 & -4.1 \\
\hline$\tau_{a a b b}$ & $(\mathrm{kHz})$ & \pm 3.2 & \pm 2.8 & \pm 1.2 \\
\hline$\tau_{a b a b}$ & $(\mathrm{kHz})$ & $-9.8 \pm 1.5$ & $-9.5 \pm 1.3$ & $-9.1 \pm 0.7$ \\
\hline \multicolumn{2}{|c|}{$\begin{array}{l}\text { Standard } \\
\text { deviation }(\mathrm{MHz})\end{array}$} & 0.045 & 0.046 & 0.047 \\
\hline
\end{tabular}
ones.

\begin{tabular}{|c|c|c|c|c|}
\hline \multicolumn{2}{|c|}{ Lines up to } & $J=10$ & $J=20$ & $J=30$ \\
\hline$A^{\prime}$ & $(\mathrm{MHz})$ & $12406.019 \pm 0.030$ & $12406.013 \pm 0.011$ & $12406.013 \pm 0.008$ \\
\hline$B^{\prime}$ & $(\mathrm{MHz})$ & $5244.445 \pm 0.008$ & $5244.456 \pm 0.005$ & $5244.455 \pm 0.003$ \\
\hline$C^{\prime}$ & $(\mathrm{MHz})$ & $3869.194 \pm 0.007$ & $3869.190 \neq 0.005$ & $3869.189 \pm 0.003$ \\
\hline$d_{J}$ & $(\mathrm{kHz})$ & $-1.15 \pm 0.81$ & $-2.11 \pm 0.33$ & $-2.17 \pm 0.21$ \\
\hline$d_{J K}$ & $(\mathrm{kHz})$ & $-32.95 \pm 13.16$ & $-22.78 \pm 6.12$ & $-22.20 \pm 3.56$ \\
\hline$d_{K}$ & $(\mathrm{kHz})$ & $-50.42 \pm 23.41$ & $-32.05 \pm 10.39$ & $-30.95 \pm 6.16$ \\
\hline$d_{W J}$ & $\left(10^{-6}\right)$ & $0.35 \pm 0.17$ & $0.57 \pm 0.07$ & $0.58 \pm 0.03$ \\
\hline$d_{W K}$ & $\left(10^{-6}\right)$ & $7.39 \pm 3.00$ & $4.89 \pm 1.39$ & $4.75 \pm 0.80$ \\
\hline \multicolumn{2}{|c|}{$\begin{array}{l}\text { Standard } \\
\text { deviation }(\mathrm{MHz})\end{array}$} & 0.047 & 0.044 & 0.039 \\
\hline
\end{tabular}

Table 1. Molecular constants from the Dowling's theory.

Table 2. Molecular constants from the Watson's theory. 
Table 3. Observed and calculated frequencies for the ground vib. state of 1.3 Propiolactone.

\begin{tabular}{|c|c|c|c|c|}
\hline $\begin{array}{l}\text { Transitior } \\
J_{K_{-1}}, K_{+1}\end{array}$ & $J^{\prime} K^{\prime}-1, K^{\prime}$ & $\begin{array}{l}\text { Observed } \\
\text { frequency } \\
(\mathrm{MHz})\end{array}$ & $\begin{array}{l}\text { Calcul ated } \\
\text { Dowling } \\
(\mathrm{MHz})\end{array}$ & $\begin{array}{l}\text { frequency } \\
\text { Watson } \\
(\mathrm{MHz})\end{array}$ \\
\hline $1_{0,1}$ & $2_{0,2}$ & 18047.65 & 18047.60 & 18047.58 \\
\hline $1_{1,1}$ & $2_{1,2}$ & 16852.03 & 16852.02 & 16852.00 \\
\hline $1_{1,0}$ & $2_{1,1}$ & 19602.46 & 19602.52 & 19602.51 \\
\hline 2,2 & $3_{1,3}$ & 25172.15 & 25172.19 & 25172.18 \\
\hline $2_{0,2}$ & $3_{0,3}$ & 26641.55 & 26641.61 & 26641.59 \\
\hline 2,0 & $3_{2,1}$ & 28040.10 & 28040.09 & 28040.08 \\
\hline $2_{2,1}$ & $3_{22}$ & 27340.86 & 27340.81 & 27340.79 \\
\hline 2,1 & $3_{1,2}$ & 29283.00 & 29283.10 & 29283.09 \\
\hline $3_{1,3}$ & $3_{2,2}$ & 27778.89 & 27778.90 & 27778.90 \\
\hline $3_{2,2}$ & $3_{3,1}$ & 39351.12 & 39351.08 & 39351.12 \\
\hline $3_{0,3}$ & $4_{0,4}$ & 34816.24 & 34816.22 & 34816.22 \\
\hline $3_{1,3}$ & $4_{1.4}$ & 33384.87 & 33384.81 & 33384.81 \\
\hline 3,2 & $4_{1,3}$ & 38797.80 & 38797.81 & 38797.81 \\
\hline $4_{1,4}$ & $5_{0,5}$ & 39572.57 & 39572.54 & 39572.59 \\
\hline $7_{2,5}$ & $7_{3,4}$ & 31347.03 & 31347.06 & 31347.03 \\
\hline $8_{1,7}$ & $8_{2,6}$ & 28231.77 & 28231.76 & 28231.75 \\
\hline $8_{2,6}$ & 83,5 & 29872.15 & 29872.12 & 29872.11 \\
\hline $9_{2,7}$ & $9_{3,6}$ & 29600.09 & 29600.11 & 29600.12 \\
\hline $10_{2,8}$ & $10_{3,7}$ & 30940.29 & 30940.26 & 30940.28 \\
\hline $12_{2,10}$ & $12_{3,9}$ & 39284.53 & 39284.61 & 39284.56 \\
\hline $12_{3,9}$ & $12_{4,8}$ & 39415.12 & 39415.14 & 39415.14 \\
\hline $13_{3,10}$ & $13_{4,9}$ & 39045.50 & 39045.45 & 39045.48 \\
\hline $15_{4,12}$ & $15_{4,11}$ & 30780.83 & 30780.80 & 30780.81 \\
\hline 185,14 & $18_{5,13}$ & 28490.59 & 28490.54 & 28490.59 \\
\hline $25_{7,19}$ & $25_{7,18}$ & 31234.30 & 31234.35 & 31234.30 \\
\hline $26_{16,10}$ & $27_{15.13}$ & 9061.01 & 9061.01 & 9061.01 \\
\hline $26_{16,11}$ & $27_{15,12}$ & 9061.01 & 9061.01 & 9061.01 \\
\hline $28_{8,21}$ & $28_{8,20}$ & 26647.50 & 26647.47 & 26647.50 \\
\hline $0_{0.0}$ & $1_{1,1}$ & $16275.50 *$ & 16275.21 & 16275.19 \\
\hline $1_{0,1}$ & $1_{1,0}$ & $8536.90 *$ & 8536.81 & 8536.81 \\
\hline $1_{01}$ & 2,0 & $24013.40 *$ & 24013.58 & 24013.55 \\
\hline 21,2 & $22_{2,1}$ & $25610.65 *$ & 25610.28 & 25610.29 \\
\hline $12_{3,10}$ & $12_{3,9}$ & $31718.28 *$ & 31718.14 & 31718.13 \\
\hline
\end{tabular}

* Not included in the fit.

\section{Discussion}

The application of Watson's treatment has allowed the observed rotational spectrum to be fit within experimental error somewhat better than Dowling's treatment. The near equivalence of the two calculations can be tested by transforming the rotational constants of Table 1 to the Watson's $d_{j}$ using Dowling's relations, together with Eq. (36) from Ref. ${ }^{3}$ and Eq. (7) from Reference ${ }^{11}$. The

Table 4. Calculated d's from Table $1 \tau$ 's.

\begin{tabular}{|c|c|c|c|}
\hline Lines up to & $J=10$ & $J=20$ & $J=30$ \\
\hline$d_{J} \quad(\mathrm{kHz})$ & $-\quad 1.15$ & $-\quad 1.47$ & $-\quad 1.46$ \\
\hline$d_{J K}(\mathrm{kHz})$ & -32.75 & -34.08 & -32.97 \\
\hline$d_{K} \quad(\mathrm{kHz})$ & -50.10 & -50.75 & -48.74 \\
\hline$d_{W J}\left(10^{-6}\right)$ & 0.36 & 0.46 & 0.47 \\
\hline$d_{W K}\left(10^{-6}\right)$ & 7.35 & 7.42 & 7.18 \\
\hline
\end{tabular}

calculations for each step are shown in Table 4. The good agreement between the two formalisms for transitions with $J \leqq 10$ deteriorates when lines of higher $J$ are included in the fit, so we think that the planarity contraints couldn't be applied. Another precaution has been taken to check the validity for the adopted constants: $1^{\circ}$ ) the constants which result from the fitting should be able to predict measured lines which are not included in analysis. For instance a "prediction" of the ground state transitions of Ref. ${ }^{40}$ showed that deviation between the predicted and observed transition frequencies are for each case within their experimental error. $2^{\circ}$ ) Previously unreported lines $(Q$ and $R$ ) were predicted (see Table 5). The Q lines are clearly

Table 5. Predicted lines included in the last fit to refine results.

\begin{tabular}{llll}
\hline \multicolumn{2}{l}{ Transition } & \multicolumn{2}{l}{ Frequencies $(\mathrm{MHz})$} \\
$J_{K-1}, K_{+1}$ & $J_{K^{\prime}-1}^{\prime}, K^{\prime}+1$ & Predicted & Observed \\
\hline $11_{6,6}$ & $12_{5,7}$ & 28237.69 & $28237.75^{*}$ \\
$13_{7,7}$ & $14_{6,8}$ & 30926.87 & $30926.81^{*}$ \\
$14_{4,11}$ & $14_{4,10}$ & 21923.08 & 21923.06 \\
$17_{5,13}$ & $17_{5,12}$ & 19757.34 & 19757.31 \\
$19_{5,15}$ & $19_{5,14}$ & 38776.26 & 38776.28 \\
$21_{6,16}$ & $21_{6,15}$ & 25300.76 & 25300.75 \\
$20_{12,8}$ & $21_{11,11}$ & 16205.37 & $16205.41^{*}$ \\
$20_{12,9}$ & $21_{11,10}$ & 16205.46 & $16205.41^{*}$ \\
$24_{7,18}$ & $24_{7} 17$ & 21650.94 & 21650.90 \\
$27_{8,20}$ & $27_{8,19}$ & 17928.33 & 17928.31 \\
\hline
\end{tabular}

* Estimated experimental error $\leqq 0.1 \mathrm{MHz}$.

found in our records with deviations between predicted and observed frequencies within our experimental error. For the contrary, the $\mathrm{R}$ lines are by far more difficult to measure and their experimental error is higher than the $Q$ ones. These lines should be included in the fit in order to refine the calculated values of the rotational constants. New values and new standard deviations are reported in

Table 6. Molecular constants from Dowling's theory (including Table 5 lines).

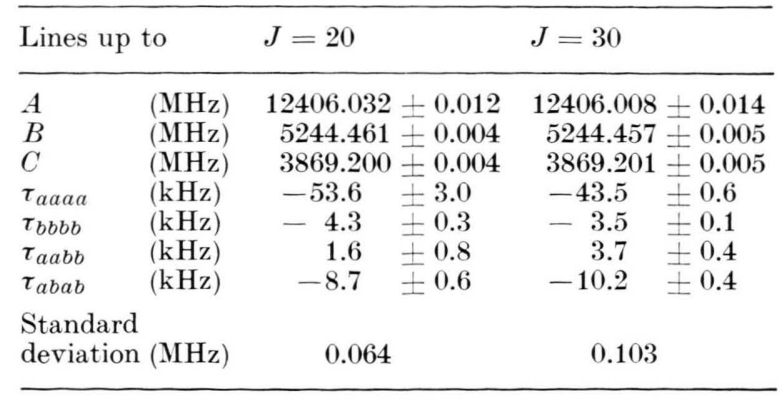


Table 7. Molecular constants from Watson's theory (including Table 5 lines).

\begin{tabular}{lrrrr}
\hline Lines up to & $J=20$ & $J=30$ \\
\hline$A^{\prime}$ & $(\mathrm{MHz})$ & $12406.006 \pm 0.009$ & $12406.012 \pm 0.005$ \\
$B^{\prime}$ & $(\mathrm{MHz})$ & $5244.455 \pm 0.003$ & $5244.456 \pm 0.002$ \\
$C^{\prime}$ & $(\mathrm{MHz})$ & $3869.189 \pm 0.003$ & $3869.191 \pm 0.002$ \\
$d_{J}$ & $(\mathrm{kHz})$ & $-2.17 \pm 0.12$ & $-2.08 \pm 0.05$ \\
$d_{J K}$ & $(\mathrm{kHz})$ & $-22.89 \pm 1.87$ & $-23.63 \pm 0.95$ \\
$d_{K}$ & $(\mathrm{kHz})$ & $-32.77 \pm 3.11$ & $-33.43 \pm 1.69$ \\
$d_{W J}$ & $\left(10^{-6}\right)$ & $0.57 \pm 0.02$ & $0.56 \pm 0.01$ \\
$d_{W K}\left(10^{-6}\right)$ & $4.92 \pm 0.42$ & $5.08 \pm 0.22$ \\
\multicolumn{5}{l}{$\begin{array}{l}\text { Standard } \\
\text { deviation }(\mathrm{MHz})\end{array}$} \\
\hline
\end{tabular}

Tables 6 and 7 . Transforming again the $\tau$ 's into the $d$ 's the agreement deteriorates even more (see Table 8). Summing up, even if the statistical ranges of the standard deviations of both methods have a partial overlap we conclude that the Watson theory is acceptable in this case for the following reasons:

1 E. B. Wilson, Jr., and J. B. Howard, J. Chem. Phys. 4, 260 [1936].

2 E. B. Wilson, Jr., J. Chem. Phys. 5, 617 [1937].

3 D. Kivelson and E. B. Wilson, Jr., J. Chem. Phys. 20, 1575 [1952].

${ }^{4}$ D. Kivelson and E. B. Wilson, Jr., J. Chem. Phys. 21. 1229 [1953].

5 W. B. Olson and H. C. Allen, Jr., J. Res. Nat. Bur. Stand. A 67, 359 [1963].

${ }^{6}$ K. T. Chung and P. M. Parker, J. Chem. Phys. 38, 8 [1963].

7 K. T. Chung and P. M. Parker, J. Chem. Phys. 43, 3865 [1965].

8 K. T. Chung and P. M. Parker, J. Chem. Phys. 43, 3869 [1965].

9 F. X. Kneizys, J. N. Freedman, and S. A. Clough, J. Chem. Phys. 44, 2552 [1966].

10 K. K. Yallabandi and P. M. Parker, J. Chem. Phys. 49, 410 [1968].

11 J. K. G. Watson, J. Chem. Phys. 45, 1360 [1966].

12 J. K. G. Watson, J. Chem. Phys. 46. 1935 [1967].

13 J. K. G. Watson, J. Chem. Phys. 48, 181 [1968].

14 J. K. G. Watson, J. Chem. Phys. 48. 4517 [1968].

15 D. Kivelson, J. Chem. Phys. 22, 904 [1954].

16 L. Pierce, J. Chem. Phys. 24, 139 [1956].

17 L. Pierce, N. Di Cianni, and R. H. Jackson, J. Chem. Phys. 38. 730 [1963].

18 M. G. Pillai and R. F. Curl, Jr., J. Chem. Phys. 37, 2921 [1962].

19 R. L. Cook, J. Chem. Phys. 42, 2927 [1965].

20 A. M. Mirri and E. Mazzariol, Spectrochim. Acta 22, 785 [1966]. a) The behavior of the standard deviation with the inclusion of lines of higher $J$ (Tables 1, 2, 6 and 7);

b) better agreement between observed and calculated lines, predicted from $d$ 's of Watson model (see Table 5):

c) disagreement between the experimental $d$ 's and the calculated ones from $\tau$ 's, even more when predicted lines were included (see Table 8 ).

Table 8. Calculates d's from Table $6 \tau$ 's.

\begin{tabular}{lrr}
\hline Lines up to & $J=20$ & \multicolumn{1}{l}{$J=30$} \\
\hline$d_{J}(\mathrm{kHz})$ & -1.49 & -1.45 \\
$d_{J K}(\mathrm{kHz})$ & -31.80 & -35.91 \\
$d_{K}(\mathrm{kHz})$ & -44.68 & -54.83 \\
$d_{W J}\left(10^{-6}\right)$ & 0.49 & 0.44 \\
$d_{W K}\left(10^{-6}\right)$ & 6.88 & 7.86 \\
\hline
\end{tabular}

21 A. M. Mirri and A. Guarnieri, Spectrochim. Acta A 23, 2159 [1967].

22 R. L. Cook and W. H. Kirchhoff, J. Chem. Phys. 47, 4521 [1967].

${ }^{23}$ G. E. Herberch, R. H. Jackson, and D. J. Millen, J. Chem. Soc. A 1966, 336 .

24 A. M. Mirri, F. Scappini, L. Innamorati, and P. Favero, Spectrochim. Acta A 25, 1631 [1969].

25 V. M. Rao and R. F. Curl, Jr., J. Chem. Phys. 45, 2032 [1966].

26 G. Steenbeckeliers, Ann. Soc. Sci. Brux. 82, 331 [1968].

27 L. Pierce, R. Nelson, and C. Thomas, J. Chem. Phys. 43, 3423 [1965].

28 G. O. Sorensen, J. Mol. Spectrosc. 22, 325 [1967].

29 B. P. van Eijck, J. Mol. Spectrosc. 27. 55 [1968].

30 D. den Engelsen, J. Mol. Spectrosc. 30, 474 [1969].

31 G. Winnewisser. J. Chem. Phys. 56. 2944. [1972].

32 P. Helminger, R. L. Cook, and F. C. DeLucia, J. Chem. Phys. 56, 4581 [1972].

83 D. J. Finnigan, A. P. Cox, A. H. Brittain, and J. G. Smith, Trans. Faraday Soc. II 63, 548 [1972].

34 P. Christen, A. Bauder, and Hs. H. Günthard, J. Mol. Spectrosc. 43. 1 [1972].

35 H. Takeo and R. F. Curl, Jr., J. Mol. Spectrosc. 43, 21 [1972].

${ }^{36}$ K. M. Marstokk and H. Møllendal, J. Mol. Structure 15, 137 [1973].

37 W. H. Kirchhoff, J. Mol. Spectrosc. 41, 333 [1972].

38 L. M. Boggia. P. Favero, and O. M. Sorarrain, Chem. Phys. Letters 12, 382 [1971].

39 J. M. Dowling, J. Mol. Spectrosc. 6, 550 [1961].

40 D. W. Boone, C. O. Britt, and J. E. Boggs, J. Chem. Phys. 43, 1190 [1965]. 